"specialists" who treated sciatica with operation could be done as an outpatient iontophoresis of the leg, unaware (as I found procedure. out in speaking to them, for I speak Russian) of the rudiments of pathophysiology. The same applied to amyotrophic lateral sclerosis, to diabetic nephropathy, and a host of other maladies (including coronary disease) about which their level of knowledge is unbelievably poor. While the peaks of Russian medicine are very high there is no doubt in my mind (and in that of several of my colleagues quite "inclined" to the communist regime for political reasons) that Soviet medicine is at least two decades behind our Western level.

To sum up, while the delivery of public health is good behind the iron curtain I still am firmly convinced that should anyone need medical care he will be better off to cope with the inherent deficiencies of the American or British systems than to receive the generous, magnanimous, etc. (I am looking for adjectives to extol it) Soviet medical care.-I am, etc.,

Mardoqueo I. Salomon Clinical Professor of Medicine

New York Medical College,

\section{Endovenous Diathermy Destruction of Internal Saphenous}

SIR,-More than two years ago, believing the conventional stripping operation for varicose veins to be a rather imprecise procedure with certain hazards, we began a technique for destroying the internal saphenous vein throughout its length from within the lumen by diathermy coagulation of the intima. This eliminated the difficulties and dangers produced by the blind process when the vein was pulled off the tributaries. We are about to begin a long-term survey of the value of this treatment, but the encouraging results so far prompt me to make an initial report in the hope that others may try the method.

The technique is somewhat similar to that for an ordinary stripping operation. The internal saphenous is ligated in the groin and all tributaries both into the internal saphenous and
directly into the common femoral vein at this site are carefully ligated and divided. A specially designed electrode is then inserted into the internal saphenous vein at the ankle, where it is exposed by a small vertical incision over the internal malleolus. The "stripper" is passed the full length of the vein and brought out through the top of the divided saphenous in the groin. A ligature held around each end prevents leakage of blood. A bare area on the upper end of the insulated stripper is then connected to the diathermy machine. The stripper is then pulled up until the distal end is about half an inch $(1.2 \mathrm{~cm})$ within the vein and the lowermost catgut suture is tied fully. The leg is then raised to above the heart level so that blood is drained out of the veins as completely as possible. The electrode is now withdrawn upwards and the diathermy operated. We have found a steady rate of about one inch $(2.5 \mathrm{~cm})$ per second best, using the diathermy current employed for coagulation of vessels in other surgical operations. A much stronger current causes irritation of neighbouring nerves and muscles and is unnecessary. After the stripper has been finally withdrawn in the groin the upper ligature is also tied and the wounds closed. Bandaging is unnecessary, and the patient may walk around on the day after the operation. We prefer to keep the patients in hospital for two to three days but, since there is no bruising and little discomfort in the legs, this is not absolutely necessary. Probably the
At first we did a conventional st:ipping operation on one side and the diathermy procedure on the other. Follow-up has shown no significant difference in the results, except that diathermy is much less distressing and is a much more precise procedure. Any smal residual tributaries after two months are dealt with by sclerosant injections in the usual fashion. Pressure pads and bandages are not needed for this

The strippers we have used are made of Fluon-coated wire of a multi-strand type. There is a bare segment near the upper end, which is introduced first, and this is also rounded with a metal tip. At the lower end there are multiple small points from the bared ends of the wire. The use of Fluon makes passage of these instruments much easier than that of conventional metal or plastic strippers, and intermediate incisions to enable the stripper to negotiate the full length of the vein are rarely necessary. We hope it will soon be possible for the electrodes to be purchased cheaply in a disposable form.-I am, etc.,

General Hospital,

Birmingham

G. T. WATtS

\section{Urinary Tract Infection in the Newborn}

SIR,-It was interesting to read your leading article on urinary tract infection in the newborn (2 September, p. 542), and I agree with the importance of culturing the urine in any neonate who is unwell.

I should like to raise the point of suprapubic puncture of the bladder, since many people regard this as a reliable method of establishing whether a true urinary infection is present. In this unit cystometry is performed on most neonates with spina bifida, and this entails inserting a suprapubic cannula over a central stilette. On removing the stilette there is occasionally faecal material on the tip even though clear urine flowed freely. Evidently the tip of the needle had entered the rectum before returning to the bladder. This is more liable to occur if the needle is inserted just above the pubis, specially if directed downwards, as the bladder of a neonate is truly abdominal, the pelvis containing only the bladder neck and urethra.

In such circumstances an infected urine might reflect the rectal flora rather than infection within the bladder. I hasten to add that none of the babies in whom this occurred suffered any untoward effects. As a precaution against introducing infection neomycin $(0.2 \%)$ is instilled into the bladder at the end of the procedure.-I am, etc.,

\section{The Children's Hospital,}

Gareth G. Thomas

\section{Twenty-five Years Ago}

SIR,-A colleague of mine has brought to my notice that you have republished (Supplement, 9 September, p. 162) a letter I wrote to the B.M.f. 25 years ago. The substance of that letter is true today, I fearif not truer.

A patient today, after considering many aspects, elects to join a certain doctor's list When he or she finds that a visit or consultation is required then the doctor of their choice "is not on duty," and after being seen by a number of "duty doctors," none of whom know anything about the patient and in some cases give the impression that they care less, it may be years before they are ever fortunate enough to meet their own doctor. This is the basis of most of the complaints about general practice that I hear today.

These conditions make a great loss of confidence for the patient and a tendency to disinterest for the general practitioner. In fact, in the latter case if it were not for efficient secretaries and receptionists many G.P.s would hardly know the names of some of their registered patients. leave alone know anything about their background.

The old family doctor ceased to exist when he himself ceased to "live over the shop," and in my opinion this was the end of general practice once and for all. I am very glad I am now retired from it, though still doing a certain amount of medical work. It may be of interest to know that one of my partners referred to in the original letter still goes strong at the age of 88.-I am, etc.

Reginald H. Little Ringwood, Hants

\section{Clinical Assessment Scheme}

SIR,-I would like to reply to Mr. R. S. Murley's comments on the clinical assessment scheme (2 September, p. 592) and to refute his suggestion that the scheme is unworkable.

Firstly, assessment is not designed to be more than a rough measure of competence or incompetence. After a month it should not be difficult for a doctor to judge if a doctor is fit to practise. Secondly, the difficulty of uniform standards is common to all examination systems, but if the purpose is primarily to weed out the incompetent a sophisticated system is unnecessary. Thirdly, the accommodation issue is a subsidiary one (and not a proper criticism of the scheme). Doctors on assessment have been accommodated in Y.M.C.A. hostels and even hotels where no hospital accommodation was free. Fourthly, the consultant's admittedly arduous clinical duties (added to by the responsibility of assessments) would be still more arduous if there were fewer junior staff-the logical result if the scheme failed. Fifthlv, and lastly, hospitals employing doctors failed under the scheme are obviously on dangerous ground and those employing unregistered doctors are, of course. breaking the law. But this is evidence of authorities refusing to co-operate with the scheme rather than criticisms of its worth.

The value of the scheme to the Health Service is that it provides a practical measure of spoken English and practical competence. An E.C.F.M.G.-type examination is of little use when many Commonwealth doctors are fluent in written English and theoretical medicine but their working experience is inadequate. Hospitals find the scheme valuable because those successfully assessed are available for locum and regular posts (often those not easy to fill). The benefits to the doctor assessed are obvious. In fact the scheme is worthwhile to everyone, but some 